DOI: 10.12731/2658-6649-2020-12-4-119-130

УДК 61.616.329-002

\title{
ОБСЛЕДОВАНИЕ КАРДИОЛОГИЧЕСКИХ БОЛЬНЫХ НА ПРЕДМЕТ ВЫЯВЛЕНИЯ ГАСТРОКАРДИАЛЬНОГО СИНДРОМА РЕФЛЮКС-ЭЗОФАГИТА (ЛИТЕРАТУРНЫЙ ОБЗОР)
}

Хоробрых Т.В., Воеводина А.А.

Цель. Проанализировать литературные данные об особенностях обследования кардиологических больных на наличие у них осложнённых форм рефлюкс-эзофагита, в том числе гастрокардиального синдрома.

Заключение. В статье рассмотрены современные методы инструментальной диагностики, используемые для диагностики у кардиологического больного рефлюкс-эзофагита, осложнённого гастрокардиальным синдромом. Отмечено, что реализация дифференциального подхода в обследовании кардиологического больного на предмет выявления у него рефлюкс-эзофагита, осложнённого гастрокардиальным синдромом, возможна путём комплексного одновременного применения нескольких методов исследования. Использование комплексного сочетания методов инструментальной диагностики будут способствовать получению более содержательных и достоверных результатов, направленных на исключение вероятности несвоевременного диагностирования у кардиологических больных рефлюкс-эзофагита, осложнённого гастрокардиальным синдромом, его проявлений различного характера, от своевременности которого, в свою очередь, зависит своевременная постановка правильного диагноза, а также выбор оптимальной тактики лечения.

Ключевые слова: диагностика; метод; гастрокардиальный синдром; рефлюкс-эзофагит, обзор.

\section{EXAMINATION OF CARDIAC PATIENTS FOR THE DETECTION OF GASTROCARDIAC REFLUX-ESOPHAGITIS SYNDROME (LITERARY REVIEW)}

Khorobrikh T.V., Voevodina A.A.

Background: To analyze the literature data on the features of examination of cardiac patients for the presence of complicated forms of reflux esophagitis, including gastrocardium syndrome. 
Conclusion: The article considers modern methods of instrumental diagnostics used for the diagnosis of reflux esophagitis complicated by gastrocardium syndrome in a cardiological patient. It is noted that the implementation of a differential approach in the examination of a cardiological patient for the detection of reflux esophagitis complicated by gastrocardial syndrome is possible through the complex simultaneous application of several research methods. The use of a complex combination of instrumental diagnostic methods will contribute to obtaining more meaningful and reliable results aimed at eliminating the likelihood of untimely diagnosis in cardiac patients with reflux esophagitis complicated by gastrocardial syndrome, its manifestations of various nature, on the timeliness of which, in turn, depends on the timely correct diagnosis, as well as the choice of optimal treatment tactics.

Keywords: diagnosis; method; gastrocardial syndrome; reflux-esophagitis, review.

\section{Введение}

В настоящее время рефлюкс-эзофагит является наиболее распространённой патологией желудочно-кишечного тракта [1], которая может существовать в сочетании с иными заболеваниями пищеварительной системы, иных органов [2], а также существенно снижать качество жизни [3, 4]. К примеру, у 45-80\% больных с грыжами пищеводного отверстия диафрагмы выявляется рефлюкс-эзофагит [5]. Своевременность и правильность выявления распространённости рефлюкс-эзофагита среди населения определено медико-социальной значимостью ввиду наличия у заболевания как типичных, так и нетипичных (атипичных) симптомов, затрудняющих процесс его диагностики, одним из которых выступает гастрокардиальный синдром. Кардиалгии, возникающие у пациентов с рефлюкс-эзофагитом, нередко вызывают большие затруднения для клиницистов, которые обусловлены как сложностью проведения дифференциальной диагностики, так и не достаточным исследованием функциональной взаимообусловленности сердечно-сосудистой системы и желудочно-кишечного тракта. В научной литературе высказано мнение о наличии сочетанного характера рефлюкс-эзофагита и патологий кардии [6], в том числе и о наличии взаимосвязи гастроэзофагеальной рефлюксной болезни и ишемической болезни сердца, выражающейся в особенностях клинического течения этих заболеваний и их инструментальной диагностики [7]. Совершенно справедливо отмечено и возрастание в последние годы интереса к исследованию функциональных взаимоотношений пищевода и сердечно-сосудистой системы [8]. 
Взаимовлияние патологии сердечно-сосудистой системы и желудочно-кишечного тракта, значительно ограничивающее и затрудняющее лечебно-диагностическую тактику, а равно и широкое внедрение сердечных препаратов, достигающих положительных симптоматический эффект за счёт устранения спастической моторики пищевода и расслабления нижнего пищеводного сфинктера, фактически изменяют клиническую картину, течение и тяжесть основного заболевания. Разрешение проблемы диагностики осложнено и различными вариантами сочетания рефлюкс-эзофагита и кардиальной патологией, существующих как в виде самостоятельных заболеваний, что бывает зачастую, так и в виде патогенетически взаимосвязанных. В доктрине отмечены такие кардиальные проявления рефлюкс-эзофагита, как нарушения проводимости, транзиторные нарушения сердечного ритма, однако наиболее часто встречающимся нарушением указана экстрасистолия [9]. Указанные клинические проявления нередко вынуждают пациентов обращаться за медицинской помощью к врачам терапевтам, кардиологам для проведения симптоматической терапии в виде сердечных препаратов [10].

Вместе с тем, учитывая достаточно близкое анатомическое расположение и общность иннервации кардии и желудка, дифференциация действительных оснований возникновения загрудинной боли является нередко затруднительной.

Достижение медицинских технологий способствовали разработке новых способов диагностики данных нозоологических форм. В то же время, значительная вариативность клинических проявлений рефлюкс-эзофагита и его коморбидности зачастую не учитывается при проведении диагностики. Однако не учёт возможной вариативности проявления симптоматики рефлюкс-эзофагита может привести к фактической недооценке рисков развития таких осложнений. Более того, использование медикаментозной терапии, сочетанной с рекомендациями по соблюдению диеты или определённого образа жизни, нередко способствует лишь купированию симптомов рефлюкс-эзофагита, не устраняя его действительные причины возникновения и развития $[11,12]$. Безусловно, необходимо учитывать, что определение перспектив применения конкретных лечебных технологий поставлено в зависимость от необходимости исследования многокомпонентных патологических состояний [13].

\section{Цель исследования}

Проанализировать литературные данные об особенностях обследования кардиологических больных на наличие у них осложнённых форм рефлюкс-эзофагита, в том числе гастрокардиального синдром. 


\section{Основная часть}

В повседневной врачебной практике обоснованно приобретает широкое распространение диагностика нарушений сердечного ритма, аритмий на этиологическом, клиническом диагностическом этапах [14]. Пациентам, обращающимся с кардиологической симптоматикой, в первую очередь, выполняется электрокардиограмма. Наличие у больных с кардиологической симптоматикой симптомов гастроэзофагеального рефлюкса отражается на результатах электрокардиограммы в виде депрессии сегмента ST, желудочковой экстрасистолии [15], фибрилляции предсердий [16].

Выполнение электрокардиограммы, безусловно, не отменяет возможность проведения диагностики с помощью холтеровского мониторирования, представляющего собой неинвазивную диагностическую процедуру, проводимую с целью анализа сердечных функций, таких, как автоматизм, проводимость, рефрактерность. Данный метод инструментальной диагностики предоставляет исследователю получить наиболее «объёмную» с точки зрения содержания и достоверную информацию о частоте аритмий, позволяя установить соответствующий контроль эффективности проведённого лечения. Результаты холтеровского мониторирования позволяют установить и оценить наличие причинной связи между возникновением аритмий сердца и иными клиническими симптомами, зафиксировать их возникновение, длительность, характер и динамику, выявить причины сердечного нарушения. Практическую значимость применения этого цифрового метода исследования усматривают в более информативном содержании его результатов в сравнении с иными методами исследования, не использующих прямых оперативных вмешательств в сердечно-сосудистую систему, в том числе путём диагностирования возможных различных нарушений сердечного ритма, безболевых приступов ишемии миокарда [17]. В доктрине отмечено, что из результатов показателей болевой и безболевой ишемии миокарда, полученных в результате комплексного использования суточного холтеровского мониторирования и электрокардиограммы, усматривались существенные данные о максимальном смещении сегмента ST, продолжительности ишемии миокарда в течение суток и количестве их эпизодов, суммарной площади сегмента ST, общем количестве эпизодов [18]. В ином случае указано, что

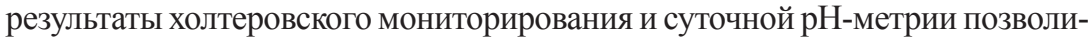
ли утверждать о возможности увеличения частоты сердечных сокращений и артериального давления при наличии у пациента гастроэзофагеального рефлюкса, симптоматика которого нередко вызывает боль за грудиной [19]. В другом исследовании, направленном на изучение функционального состо- 
яния сердечно-сосудистой системы при наличии патологии верхнего отдела желудочно-кишечного тракта, обосновано улучшение показателей проводимости, вегетативных влияний при условии «нормализации» показателей суточного рН-мониторирования, электрокардиограммы, холтеровского мониторирования [20] .

Не ставится под сомнение справедливость утверждения о возможности окончательного разрешения вопроса об определении надлежащей тактики лечения только после оценки результатов, полученных при комплексном использовании таких методов инструментальной диагностики, как суточное мониторирование внутрипищеводного $\mathrm{pH}$ и холтеровского мониторирования [21]. Возможность получения результатов исследований, исходя из применения различных методов инструментальной диагностики, фактически имеющих различное целевое назначение и задачи исследования, способствует диагностированию количественной информации, к примеру, о степени ацидификации пищевода, сопоставлении эпизодов рефлюкса с изменениями на электрокардиограмме. Кроме того, повышается вероятность верифицировать типичные и атипичные симптомы ишемической болезни сердца, нетипичным проявлением которой выступает рефлюкс-эзофагит, нередко провоцирующий возникновение этого заболевания [22]. Результаты холтеровского мониторирования также позволяют оценить наличие причинной связи между возникновением аритмий сердца и иными клиническими симптомами, зафиксировать их возникновение, длительность и динамику, выявить причины нарушения сердечного ритма.

Мультидетекторная компьютерная томография - неизвазивный метод исследования, который обоснованно признаётся высокоинформативным методом, применяемым в диагностике грыж пищеводного отверстия диафрагмы. С помощью данного метода возможно установление перемещения большой части желудка в грудную полость, диагностирование инвагинации пищевода в желудок, определение наличия недостаточности кардии, выявление признаков воспаления зоны пишеводно-желудочного перехода. В положительной динамике практического применения МДКТ-исследования отмечено, что его результаты позволяют получать трёхмерные изображения при субмиллиметровом сканировании, что фактически более расширяет границы применения этого цифрового метода исследования, возможного к использованию для изучения анатомии сердца и коронарных сосудов [23]. Более того, ввиду наличия дополнительных технических возможностей мультидетекторной компьютерной томографии, выражающихся в возможности проектирования в виде $3 \mathrm{D}$ реконструкций, результаты 
её применения фактически способствуют визуализации патологической зоны в объёмном формате [24].

Наибольшую информативную основу состояния слизистой оболочки пищевода предоставляют результаты эндоскопии, позволяющей визуально произвести оценку изменений слизистой оболочки, оценить состояние желудочно-пищеводного перехода и Z- образной линии. Это, в свою очередь, способствует визуализации кардии ретроградно, предоставляя возможность оценить замыкательную функцию кардии. Кроме того, эндокопическое исследование позволяет оценить степень сужения пищевода, визуализировать грыжу пищеводного отверстия диафрагмы.

При обследовании кардиологического больного целесообразно применение дифференциальной диагностики, что обусловлено тем, что нередко внепищеводным проявлением рефлюкс-ззофагита выступает гастрокардиальный синдром, связанный с возникновением и развитием патологии в области кардии. В свою очередь, поскольку выбор надлежащей тактики лечения больного зависит от правильной верификации действительных оснований его основного и сопутствующего заболевания, то в исследуемом случае реализация применения дифференциальной диагностики между кардиопатологией и патологией органов желудочно-кишечного тракта объективно оправданна.

\section{Заключение}

Целенаправленность диагностики выявления истинных причин проявлений патологии со стороны сердечно-сосудистой системы изначально должна иметь парадигму выявления отклонений и патологий в области кардии. При установлении несоответствия клинических проявлений и получении данных различных инструментальных исследований диагностика причинности должна «расширяться» по мере нахождения изменений не только со стороны сердечно-сосудистой системы. Безусловно, первоначальное обследование пациента с кардиологическими болями в виде применения электрокардиограммы, холтеровского мониторирования не ставится под сомнение. Однако коморбидность заболеваний сердечно-сосудистой системы и патологий пищевода не учитывать недопустимо. Именно поэтому для исключения или подтверждения наличия патологий пищевода изначально целесообразно также и использование фиброгастродуоденоскопии, а для содержательной «полноты» клинической картины оправданно и дополнение обследования путём применения мультидетекторной компьютерной томографии. Наличие информативности каждого из указанных методов обследования предполагается и не умаляется, вместе с 
тем, не исключается, что результаты, полученные при возможном «разрозненном» их применении, могут быть недостаточными для установления и определения патологических изменений, их причин и динамики развития. Применение же совокупности методов обследования способствует верификации оснований всех патологических изменений, что, безусловно, способствует своевременной постановке правильного диагноза с целью определения дальнейшей тактики лечения пациента, улучшающей качество его жизни. «Суммарная» оценка результатов, полученных при «комбинированном» сочетании и использовании приведённых методов обследования, фактически будет являться более «информативной», содержательной, с наибольшей степенью достоверности.

\section{Список литературы}

1. El-Serag H.B., Sweet S., Winchester C.C. et al. Update on the epidemiology of gastro-oesophageal reflux disease: a systematic review // Gut. 2014, №63, pp.871-80. doi: 10.1136/gutjnl-2012-304269

2. Saritas Yuksel E., Vaezi M.F. Extraesophageal manifestations of gastroesophageal reflux disease: cough, asthma, laryngitis, chest pain // Swiss Med Wkly. 2012, №142: w13544. doi: 10.4414/smw.2012.13544

3. Krenk L., Kehlen H., Hansen T. et al. Cognitive dysfunction after fast-track hip and knee replacement // Anesthesia \& Analgesia. 2014, No, 5, pp. 1034-1040. doi: 10.1213/ANE.0000000000000194

4. Geremy R., Sheraz Z., Melody Z. et al. Laparoscopic repair of hiatus hernia: Does mesh type influence outcome? A meta-analysis and European survey study // Surgical Endoscopy. 2016, Vol, 12, pp. 5209-5221. doi: 10.1007/s00464-016-4900-3

5. Мусабаев Н.Х., Имантаева Г.М., Камзина З.К., Камбаров Ф.Т., Абдулхамитова Ж.Б., Абдиев Ш.Г., Абишева А.К., Ибрагимов А.Р., Кайыржанова С.А. Профилактика кишечных свищей // Вестник КазНМУ. 2018. № 1. С. 245.

6. Алексеева О.П., Пикулев Д.В. Гастроэзофагеальная рефлюксная болезнь и ишемическая болезнь сердца - существует ли синдром взаимного отягощения? // Российский журнал гастроэнтерологии, гепатологии, колопроктологии. 2019. №29(4). С.66-73.

7. Пикулев Д.В., Алексеева О.П., Долбин И.В. Ишемическая болезнь сердца и гастроэзофагеальная рефлюксная болезнь: особенности сочетанного течения // Медальманах. 2012. №1(20). С.43-9.

8. Антропова О.Н., Пырикова Н.В., Осипова И.В. Фибрилляция предсердий и гастроэзофагеальная болезнь: механизмы взаимосвязи, подходы к лечению // Российский кардиологический журнал. 2019. №7. С.103-109. 
9. Костюкевич О.И., Рылова А.К., Рылова Н.В., Былова Н.А., Колесникова Е.А., Корсунская М.И., Симбирцева А.С. Внепищеводные маски гастроэзофагеальной рефлюксной болезни // РМЖ. Медицинское обозрение. 2018. №7(II). C. $76-81$.

10. Maradey-Romero C., Fass R. New therapies for non-cardiac chest pain // Curr Gasrtoenterol Rep. 2014, №16, pp. 390.

11. Бородин Д.С. «Кислотный карман» как патогенетическая основа и терапевтическая мишень при гастроэзофагеальной рефлюксной болезни // Терапевтический архив. 2014. № 2. С.76-80.

12. Булгаков С.А. «Гевискон» при изжоге, обусловленной гастроэзофагеальной рефлюксной болезнью // Новости медицины и фармации. 2013. № 451. С. 34-37.

13. Щепин В.О., Бравве А.И., Бабенко А.И., Томчук А.Л. Методические подходы к расчёту потенциальной востребованности клинико-диагностических технологий // Проблемы социальной гигиены, здравоохранения и истории медицины. 2013. № 5. С. 39-42.

14. Магомедова Б.М., Глебов К.Г., Теплова Н.В., Котовский А.Е., Артамонова Н.Г. Изменения слизистой оболочки верхних отделов желудочно-кишечного тракта при ишемической болезни сердца // Кардиоваскулярная терапия и профилактика. 2017. №16 (6). С. 65-69.

15. Еремина Е.Ю., Зверева С.И., Козлова Л. С. Показатели вариабельности сердечного ритма у пациентов с гастроэзофагеальной рефлюксной болезнью // Экспериментальная и клиническая гастроэнтерология. 2018. №157(9). С. 10-17.

16. Алейникова Т.В., Мистюкевич И.И. Возможности холтеровского мониторирования в диагностике заболеваний сердечно-сосудистой системы // Проблемы здоровья и экологии. 2014. № 1 (39). С. 14-20.

17. Huang CC, Chan WL, Luo JC, et al. Gastroesophageal reflux disease and atrial fibrillation: a nationwide population-based study // PLoS One. 2012, №7 (10), e47575. doi: 10.1371/journal.pone.0047575

18. Федулаев Ю.Н., Корочкин И.М., Кузьменкова Л.В. Сравнительная характеристика качественных и количественных показателей преходящей ишемии миокарда, данных суточной РН-метрии у больных с ишемической болезнью сердца и гастроэзофагеальной рефлюксной болезнью // Российский кардиологический журнал. 2011. №1. С.45-48.

19. Маев И.В., Вьючнова Е.С., Щекина М.И. Гастроэзофагеальная рефлюксная болезнь - болезнь XXI века // Лечащий врач. 2004. №4. С.10-14.

20. Кузьмина А.Ю. Состояние сердечно-сосудистой системы // Лечащий врач. 2004. №4. C.5-8.

21. Таранченко Ю.В., Звенигородская Л.А. Дифференциальная диагностика загрудинных болей при сочетании гастроэзофагеальной рефлюксной бо- 
лезни с ишемической болезнью сердца // Consilium Medicum. 2002. T. 4. № 6. C. 3-4.

22. Сторонова О.А., Трухманов А.С. 24-часовая рН-импедансометрия. Дифференциальный диагноз функциональных заболеваний пищевода. Пособие для врачей / Под ред. акад. РАН, проф. В.Т. Ивашкина М.: ИД «МЕДПРАКТИКА-М». 2018. 32 c.

23. Мин Д.К, Кох Р., Аппельбаум Д., Уанн С. Мультидетекторная компьютерная томография в диагностике сердечно-сосудистых заболеваний. Обзор литературы // Международный журнал интервенционной кардиоангиологии. 2005. № 8. С. 39-47.

24. Бурякина С.А., Ручкин Д.В., Цинь Я., Кармазановский Г.Г., Бочарникова А.В. МДКТ-семиотика послеожоговых рубцовых сужений пищевода // Медицинская визуализация. 2013. № 5. С. 62-75.

\section{References}

1. El-Serag H.B., Sweet S., Winchester C.C. et al. Update on the epidemiology of gastro-oesophageal reflux disease: a systematic review. Gut. 2014, №63, pp.871-80. doi: 10.1136/gutjnl-2012-304269

2. Saritas Yuksel E., Vaezi M.F. Extraesophageal manifestations of gastroesophageal reflux disease: cough, asthma, laryngitis, chest pain. Swiss Med Wkly. 2012, №142: w13544. doi: 10.4414/smw.2012.13544

3. Krenk L., Kehlen H., Hansen T. et al. Cognitive dysfunction after fast-track hip and knee replacement. Anesthesia \& Analgesia. 2014, No, 5, pp. 1034-1040. doi: 10.1213/ANE.0000000000000194

4. Geremy R., Sheraz Z., Melody Z. et al. Laparoscopic repair of hiatus hernia: Does mesh type influence outcome? A meta-analysis and European survey study. Surgical Endoscopy. 2016, Vol, 12, pp. 5209-5221. doi: 10.1007/s00464-0164900-3

5. Musabaev N. H., Imantaeva G. M., kamzina Z. K., Kambarov F. T., Abdulhamitova Zh. b., Abdiev sh. G., Abisheva A. K., Ibragimov A. R., Kayyrzhanova S. A. Profilaktika kishechnykh svishchey [Prevention of intestinal fistulas]. Vestnik KazNMU [Bulletin of KazNMU]. 2018, № 1, pp. 245.

6. Alekseeva O. P., Pikulev D. V. Gastroezofageal'naya reflyuksnaya bolezn' i ishemicheskaya bolezn' serdtsa - sushchestvuet li sindrom vzaimnogo otyagoshcheniya? [Gastroesophageal Reflux disease and coronary heart disease - is there a mutual burden syndrome?]. Rossiyskiy zhurnal gastroenterologii, gepatologii, koloproktologii [Russian journal of gastroenterology, Hepatology, Coloproctology]. 2019, № 29 (4), pp. 66-73. 
7. Pikulev D. V., Alekseeva O. P., Dolbin I. V. Ishemicheskaya bolezn' serdtsa i gastroezofageal'naya reflyuksnaya bolezn': osobennosti sochetannogo techeniya [Ischemic heart disease and gastroesophageal reflux disease: features of the combined course]. Medical Almanac. 2012, № 1(20), pp. 43-49.

8. Antropova O. N., Pyrikova N. V., Osipova I. V. Fibrillyatsiya predserdiy i gastroezofageal'naya bolezn': mekhanizmy vzaimosvyazi, podkhody $\mathrm{k}$ lecheniyu [Atrial Fibrillation and gastroesophageal disease: mechanisms of interrelation, approaches to treatment]. Rossiyskiy kardiologicheskiy zhurnal [Russian journal of cardiology]. 2019, № 7, pp. 103-109.

9. Kostyukevich O. I., Rylova A. K., Rylova N. V., Bylova N. A., Kolesnikova E. A., Korsunskaya M. I., Simbirtseva A. S. Vnepishchevodnye maski gastroezofageal'noy reflyuksnoy bolezni [Extraesophageal masks of gastroesophageal reflux disease]. RMZh. Meditsinskoe obozrenie [Medical review]. 2018, № 7 (II), pp. 76-81.

10. Maradey-Romero C., Fass R. New therapies for non-cardiac chest pain. Curr Gasrtoenterol Rep. 2014, № 16, pp. 390.

11. Borodin D. S. «Kislotnyy karman» kak patogeneticheskaya osnova i terapevticheskaya mishen' pri gastroezofageal'noy reflyuksnoy bolezni ["Acid pocket" as a pathogenetic basis and therapeutic target in gastroesophageal reflux disease]. Terapevticheskiy arkhiv [Therapeutic archive]. 2014, № 2, pp. 76-80.

12. Bulgakov S. A. «Geviskon» pri izzhoge, obuslovlennoy gastroezofageal'noy reflyuksnoy bolezn'yu ["Gaviscon" for heartburn caused by gastroesophageal reflux disease]. Novosti meditsiny i farmatsii [News of medicine and pharmacy]. 2013, № 451, pp. 34-37.

13. Shchepin V. O., Bravve A. I., Babenko A. I., Tomchuk A. L. Metodicheskie podkhody $\mathrm{k}$ raschetu potentsial'noy vostrebovannosti kliniko-diagnosticheskikh tekhnologiy [Methodological approaches to calculating the potential demand for clinical and diagnostic technologies]. Problemy sotsial'noy gigieny, zdravookhraneniya $i$ istorii meditsiny [Problems of social hygiene, health care and the history of medicine]. 2013, № 5, pp. 39-42.

14. Magomedova B. M., Glebov K. G., Teplova N. V., Kotovsky A. E., Artamonova N. G. Izmeneniya slizistoy obolochki verkhnikh otdelov zheludochno-kishechnogo trakta pri ishemicheskoy bolezni serdtsa [Changes in the mucous membrane of the upper gastrointestinal tract in ischemic heart disease]. Kardiovaskulyarnaya terapiya i profilaktika [Cardiovascular therapy and prevention]. 2017, № 16 (6), pp. 65-69.

15. Eremina E. Yu., Zvereva S. I., Kozlova L. S. Pokazateli variabel'nosti serdechnogo ritma u patsientov s gastroezofageal'noy reflyuksnoy bolezn'yu [Indicators of heart rate variability in patients with gastroesophageal reflux disease]. Eks- 
perimental'naya i klinicheskaya gastroenterologiya [Experimental and clinical gastroenterology]. 2018, №157 (9), pp. 10-17.

16. Aleynikova T. V., Mistyukevich I. I. Vozmozhnosti kholterovskogo monitorirovaniya $\mathrm{v}$ diagnostike zabolevaniy serdechno-sosudistoy sistemy [Possibilities of Holter monitoring in diagnostics of diseases of the cardiovascular system]. Problemy zdorov'ya i ekologii [Problems of health and ecology]. 2014, № 1 (39), pp. 14-20.

17. Huang CC, Chan WL, Luo JC, et al. Gastroesophageal reflux disease and atrial fibrillation: a nationwide population-based study. PLoS One. 2012, №7 (10), e47575. doi: 10.1371/journal.pone.0047575

18. Fedulaev Yu. N., Korochkin I. M., kuzmenkova L. V. Sravnitel'naya kharakteristika kachestvennykh i kolichestvennykh pokazateley prekhodyashchey ishemii miokarda, dannykh sutochnoy PH-metrii u bol'nykh s ishemicheskoy bolezn'yu serdtsa i gastroezofageal'noy reflyuksnoy bolezn'yu [Comparative characteristics of qualitative and quantitative indicators of transient myocardial ischemia, daily PH-metry data in patients with coronary heart disease and gastroesophageal reflux disease]. Rossiyskiy kardiologicheskiy zhurnal [Russian journal of cardiology]. 2011, № 1, pp. 45-48.

19. Mayev I. V., Vyuchnova E. S., Shchekina M. I. Gastroezofageal'naya reflyuksnaya bolezn' - bolezn' XXI veka [Gastroesophageal Reflux disease - a disease of the XXI century]. Lechashchiy vrach [Attending physician]. 2004, № 4, pp. 10-14.

20. Kuzmina A. Yu. Sostoyanie serdechno-sosudistoy sistemy [State of the cardiovascular system]. Lechashchiy vrach [Attending physician]. 2004, № 4, pp. 5-8.

21. Taranchenko Yu. V., Zvenigorodskaya L. A. Differentsial'naya diagnostika zagrudinnykh boley pri sochetanii gastroezofageal'noy reflyuksnoy bolezni $\mathrm{s}$ ishemicheskoy bolezn'yu serdtsa [Differential diagnostics of chest pains in combination of gastroesophageal reflux disease with ischemic heart disease]. Consilium Medicum. 2002, Vol, 4, № 6. pp. 3-4.

22. Storonova O. A., Trukhmanov A. S. 24-chasovaya rN-impedansometriya. Differentsial'nyy diagnoz funktsional'nykh zabolevaniy pishchevoda. Posobie dlya vrachey [24-hour $\mathrm{pH}$-impedance measurement. Differential diagnosis of functional diseases of the esophagus. Manual for doctors] / ed. Acad. Russian Academy of Sciences, Professor V. T. Ivashkin. M.: publishing house "MEDPRAKTIKA-M". 2018, 32 p.

23. Min D. K, Koch R., Appelbaum D., Uann S. Mul'tidetektornaya komp'yuternaya tomografiya $\mathrm{v}$ diagnostike serdechno-sosudistykh zabolevaniy. Obzor literatury [Multidetector computed tomography in the diagnosis of cardiovascular diseases. Literature review]. Mezhdunarodnyy zhurnal interventsionnoy kardioangiologii [International journal of interventional Cardioangiology]. 2005, № 8, pp. 39-47. 
24. Buryakina S. A., Ruchkin D. V., Qin Ya., Karmazanovsky G. G., Bocharnikova A.V. MDKT semiotika posleozhogovykh rubtsovykh suzheniy pishchevoda [MDKT semiotics of post-burn cicatricial narrowing of the esophagus]. Meditsinskaya vizualizatsiya [Medical imaging]. 2013, №5, pp. 62-75.

\section{ДАННЫЕ ОБ АВТОРАХ}

Хоробрых Татьяна Витальевна, д.м.н., профессор кафедры Факультетской хирургии №1 Федеральное государственное автономное образовательное учреждение выстего образования Первый Медииинский государственный медицинский университет имени И.М. Сеченова Министерства здравоохранения Российской Федерации (Сеченовский Университет)

ул. Трубецккая, 8, стр. 2, г. Москва, 119991, Российская Федераџия horobryh68@list.ru

Воеводина Анна Александровна, аспирант кафедры Факультетской хирургии №1

Федеральное государственное автономное образовательное учреждение высшего образования Первый Медицинский государственный медицинский университет имени И.М. Сеченова Министерства здравоохранения Российской Федераиии (Сеченовский Университет)

ул. Трубецкая, 8, стр. 2, г. Москва, 119991, Российская Федераџия anny1608@mail.ru

\section{DATA ABOUT THE AUTHORS}

Khorobrykh Tatyana Vitalievna, MD, Professor of the Department of Faculty Surgery №1

Sechenov University

8/2, Trubetskaya str., Moscow, 119991, Russian Federation horobryh68@list.ru

Voevodina Anna Alexsadrovna, Post-Graduate Student of the Department of Faculty Surgery №1

Sechenov University

8/2, Trubetskaya str., Moscow, 119991, Russian Federation anny1608@mail.ru 